\title{
STRUCTURAL CHARACTERIZATION OF DOPED THICK GaInNAs LAYERS — AMBIGUITIES AND CHALLENGES
}

\author{
Damian Pucicki * Katarzyna Bielak ${ }^{*}$ - Beata Ściana * \\ Wojciech Dawidowski ${ }^{*}$ - Karolina Żelazna ${ }^{* *}$ \\ Jarosław Serafińczuk ${ }^{*}$ - Jaroslav Kováćc** \\ Andrej Vincze $^{* * * *}$ — Łukasz Gelczuk ${ }^{*}$ — Piotr Dłużewski ${ }^{* * * * *}$
}

\begin{abstract}
GaInNAs alloys are mostly used as an active part of light sources for long wavelength telecom applications. Beside this, these materials are used as thin quantum wells (QWs), and a need is to grow thick layers of such semiconductor alloys for photodetectors and photovoltaic cells applications. However, structural characterization of the GaInNAs layers is hindered by non-homogeneity of the In and $\mathrm{N}$ distributions along the layer. In this work the challenges of the structural characterization of doped thick GaInNAs layers grown by atmospheric pressure metalorganic vapour phase epitaxy (APMOVPE) will be presented.
\end{abstract}

K e y w or d s: dilute nitrides, GaInNAs, composition determination, HRXRD

\section{INTRODUCTION}

Dilute nitrides have been intensively developed since 1992, when Weyers et al discovered the strong effect of redshift by adding a small amount of nitrogen to GaAs [1]. In 1996 Kondow et al demonstrated the GaInNAs as a material which could be grown coherently on GaAs. Those semiconductor materials allow rapid development of the so-called long-wavelength GaAs-based optoelectronics [2]. In 1999 GaInNAs was proposed as one of the materials for high-voltage multi-junction GaAs-based solar cells application [3]. The technology of multijunction solar cells with several subcells made of different multicomponent semiconductors requires the epitaxial growth of thick lattice matched materials, one after another. Simultaneously, the materials used for solar cells constructions must be doped in order to create $\mathrm{p}-\mathrm{n}$ junctions. The technology of the devices which contain layers made of multicomponent semiconductor alloys has to be supported by precise characterization of the grown structures.

\section{AMBIGUITIES OF COMPOSITION DETERMINATION}

Determination of the nitrogen and indium contents in GaInNAs layers is often based on high resolution X-ray diffraction (HRXRD) measurements. Taking into account numerous factors, this method is insufficient for precise composition determination of such layers. First of all, by adding nitrogen into InGaAs alloy the residual compressive strains corresponding to the presence of In are com- pensated by nitrogen related tensile strains. In agreement with Vegard's law this situation leads to many different compositions of GaInNAs alloys with the same lattice constants [4] as shown in Fig. 1.



Fig. 1. Different combinations of indium and nitrogen contents for several GaInNAs lattice constants

There are many ways of nitrogen incorporation into InGaAsN layers leading to overestimation of the nitrogen content. As discussed by $\mathrm{Li}$ et al there are adverse conditions which lead to formation of N-N or N-As complexes, due to their high formation energy in the lattice, which causes less tensile strain than the presence of substitutional $\mathrm{N}_{\mathrm{As}}$ atoms. That leads to considerable deviations from Vegard's law, which is the base of HRXRD measurements analysis [5]. The third factor providing deviation is a gradient profile of the indium and nitrogen

\footnotetext{
* Faculty of Microsystem Electronics and Photonics, Wrocław University of Technology, Z. Janiszewskiego 11/17, 50-372, Wrocław, Poland, damian.pucicki@pwr.edu.pl; ${ }^{* *}$ Institute of Physics, Wrocław University of Technology, Wyb. Wyspiańskiego 27, 50-370, Wrocław, Poland; *** Faculty of Electrical Engineering and Information Technology, Slovak University of Technology, Ilkovičova 3 , 81219 Bratislava, Slovakia; ${ }^{* * * *}$ International Laser Centre, Ilkovičova 3, 84104 Bratislava, Slovakia; ${ }^{* * * *}$ Institute of Physics, Polish Academy of Science, Al. Lotników 32/46, 02-668 Warsaw, Poland;
} 


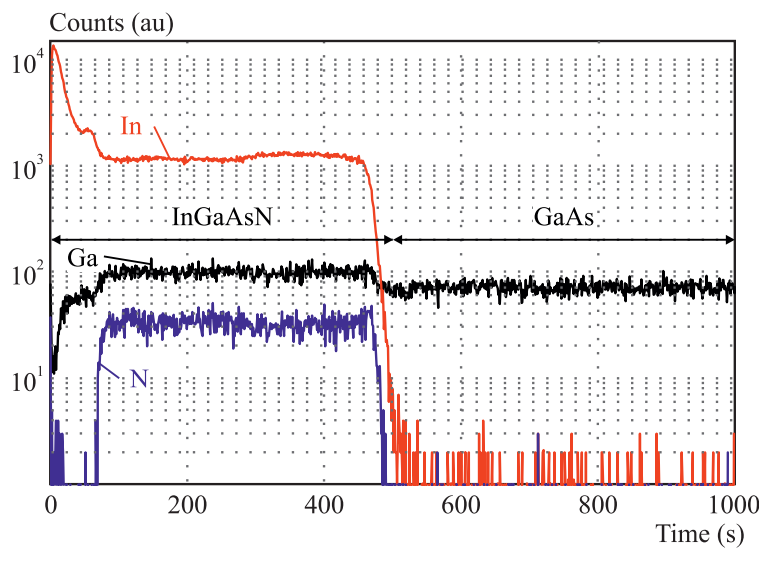

Fig. 2. SIMS profiles of the \#NI52 GaInNAs/GaAs undoped heterostructures with $\sim 110 \mathrm{~nm}$ thick inhomogeneous GaInNAs layer

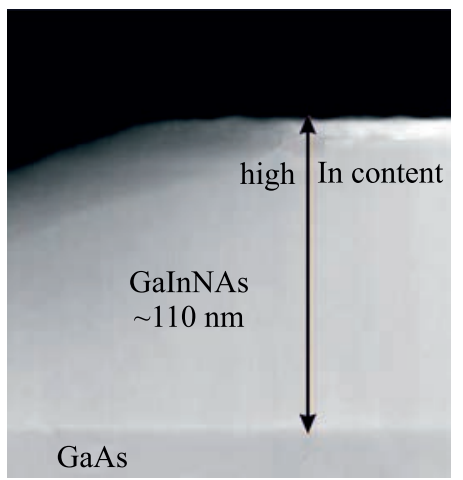

Fig. 3. Dark field TEM image of the \#NI52 GaInNAs/GaAs undoped heterostructures with a $\sim 110 \mathrm{~nm}$ thick inhomogeneous GaInNAs layer

contents along the layer as discussed in [6] and observed also on the secondary ion mass spectrometry (SIMS) profiles and transmission electron microscopy (TEM) images of investigated GaInNAs layers (Figs. 2 and 3). All of the above mentioned factors have to be considered during HRXRD analysis of thick and also doped structures containing GaInNAs layers.
Table 1. Structural parameters of the \#NI46n GaInNAs/GaAs MQW sample

\begin{tabular}{cccc}
\hline & \multicolumn{3}{c}{ Gradient QWs } \\
& $\operatorname{In}(\%)$ & $\mathrm{N}(\%)$ & $d(\mathrm{~nm})$ \\
\hline GaAs barier & - & - & 28.0 \\
\hline convex & 25.3 & 0.1 & \\
gradient & $\uparrow$ & $\uparrow$ & 18.1 \\
QW & 8.3 & 0.5 & \\
\hline GaAs barier & - & - & 28.0 \\
\hline \multicolumn{4}{c}{ Bottom of the structure } \\
\hline
\end{tabular}

3 EXPERIMENTAL DETAILS

The investigated heterostructures were grown by atmospheric pressure metalorganic vapour phase epitaxy (APMOVPE) with AIX200 R\&D AIXTRON horizontal reactor on (100)-oriented semi-insulating (SI) GaAs or Si-doped n-type GaAs substrates. Trimethylgallium (TMGa), trimethylindium (TMIn), tertiarybutylhydrazine (TBHy) and arsine $\left(\mathrm{AsH}_{3}: 10 \%\right.$ mixture in $\left.\mathrm{H}_{2}\right)$ were used as the growth precursors. High purity hydrogen was employed as a carrier gas. The GaInNAs layers were doped by diethylzinc (DEZn) and silane $\left(\mathrm{SiH}_{4}: 20 \mathrm{ppm}\right.$ in $\mathrm{H}_{2}$ ) as p-type and n-type dopant sources, respectively. The grown structures consist of 360 to $370 \mathrm{~nm}$ doped GaAs buffer layer and a 60 to $120 \mathrm{~nm}$ thick n-type or p-type GaInNAs layer.

Structural characterization of the grown GaInNAs/GaAs structures was carried out in accordance with the algorithm (Fig. 4) proposed by the authors in the previous work [4]. The proposed algorithm (Algorithm 1) couples the HRXRD measurements with theoretical calculation and determination of band gap of the investigated GaInNAs layers from the results of various optical measurements like photoluminescence (PL), contactless electroreflectance (CER), photoreflectance (PR), photovoltaic (PVS) or photocurrent (PC) spectroscopy. The

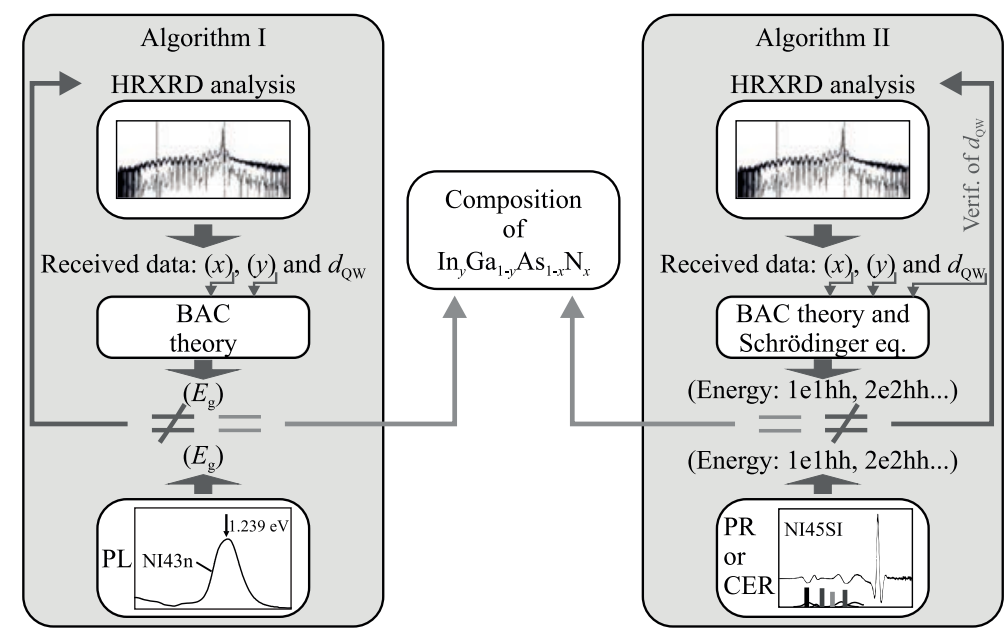

Fig. 4. Algorithms of structural characterization of GaInNAs/GaAs QWs structures 


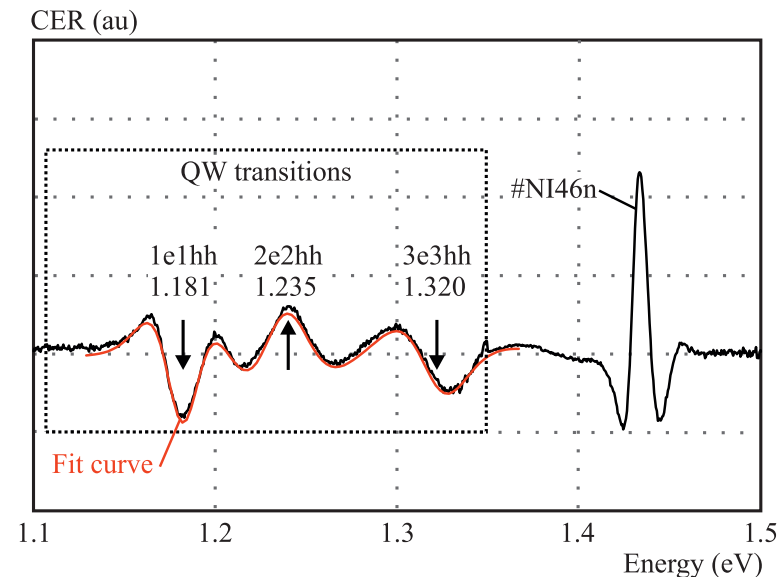

(a)

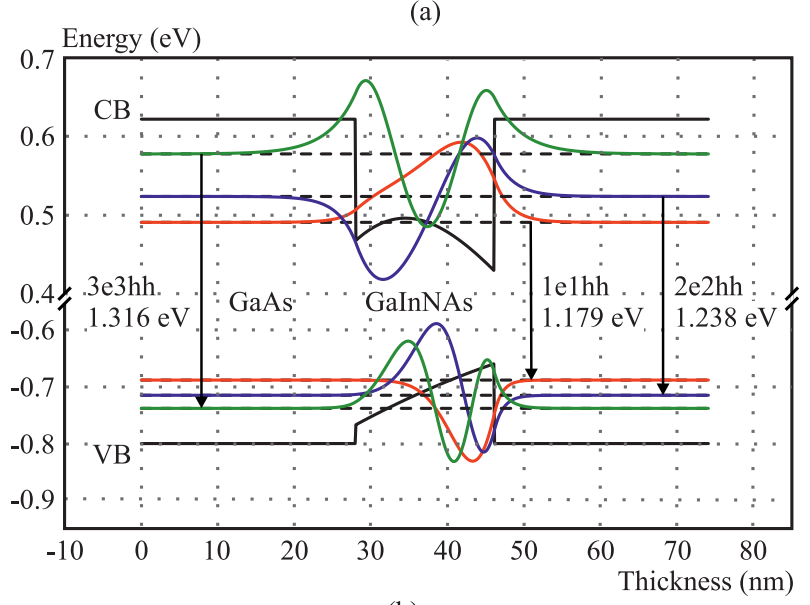

(b)



(c)

Fig. 5. The results of structural characterization of sample \#NI46n: a - CER spectrum with assigned main transitions energy, b - calculated band diagram of the non-uniform gradient QW, c-(004) $\omega / 2 \theta$ diffraction curves with results of simulations assuming the nonuniform QWs in the structure

application of the mentioned algorithm for characterization of the GaInNAs quantum wells (QWs), together with calculation and determination of all QWs transitions from CER or PR resonances (Algorithm 2), makes the proposed algorithm more accurate. In this solution, the calculation of QWs energy states separation allows characterization of GaInNAs QWs with gradient of composition or step-like QWs caused by phase segregation in GaInNAs $[4,7]$.
A sample of characterization results of such a nonhomogeneous GaInNAs MQW structure is presented in Fig. 5. In the case of \#NI46n structure with three GaInNAs/GaAs quantum wells, the applied algorithm allows to achieve convergence of the HRXRD simulation with omega/2theta scan and calculated transition energies with CER resonances. As a result of that characterization, the structural parameters of \#NI46n structures were determined more accurately than on the basis of only HRXRD analysis. The achieved parameters of these structures are presented in Tab. 1. The collected data describe all three QWs in the structure. The bottom side of the structure is assigned in the table.

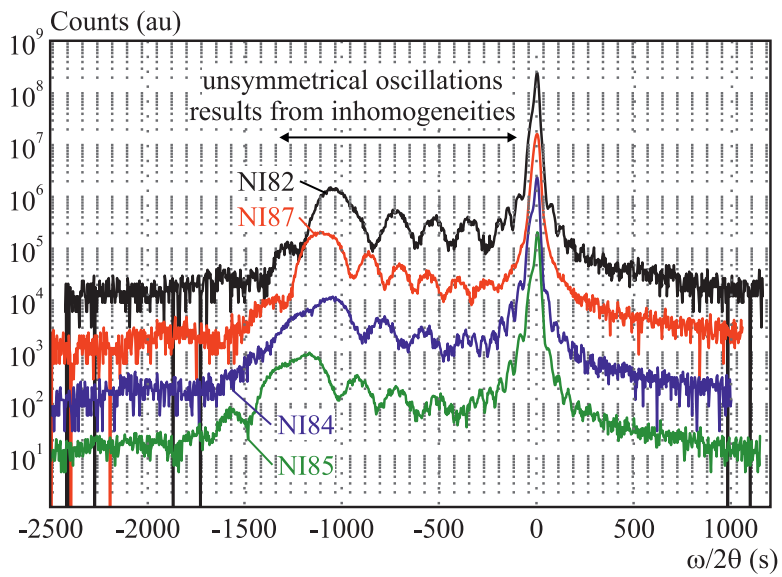

Fig. 6. The (004) $\omega / 2 \theta$ diffraction curves of the doped thick $\mathrm{p}^{+}$-GaInNAs/GaAs:Zn (\#NI82 and \#NI87) and $\mathrm{n}^{+}$-GaInNAs/Ga As:Si (\#NI84 and \#NI85) structures

\section{LIMITATION OF THE APPLIED ALGORITHM}

As it was mentioned, for structural characterization of the homogenous GaInNAs QWs or thick layers it is sufficient to couple the HRXRD analysis with the GaInNAs energy gap value. The assumption of homogeneity of the grown GaInNAs layer is not valid since the TEM images and SIMS measurements have been performed (Figs. 2 and 3). The inhomogeneity of the investigated GaInNAs layers is also observed on the diffraction curves in the form of unsymmetrical distribution of the diffraction peaks (Figs. 5c and 6).

There are several limitations of the applied structural characterization algorithm occurring in the case of characterization of the thick doped inhomogeneous GaInNAs layers. PL, PVS or PC measurements provide the averaged information about the energy gap of the GaInNAs layers resulting in a broadening of the PL spectrum or absorption edges of the investigated material. Simultaneously, there are no discreet energy subbands in the thick layers, distinct from QWs, which could results in separate resonances at the CER or PR spectra (Fig. 7). Additionally, the optical response of the doped GaInNAs layers is affected by the presence of dopant atoms in the lattice or by the presence of the built-in electrical potential across 


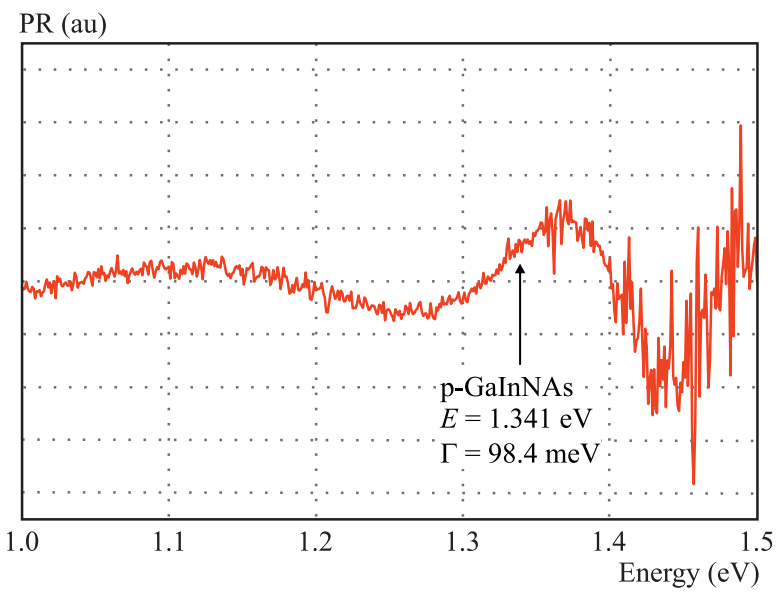

Fig. 7. The PR spectrum of the samples \#NI82 containing $\mathrm{p}^{+}$-InGaAsN $\sim 100 \mathrm{~nm}$ thick layer

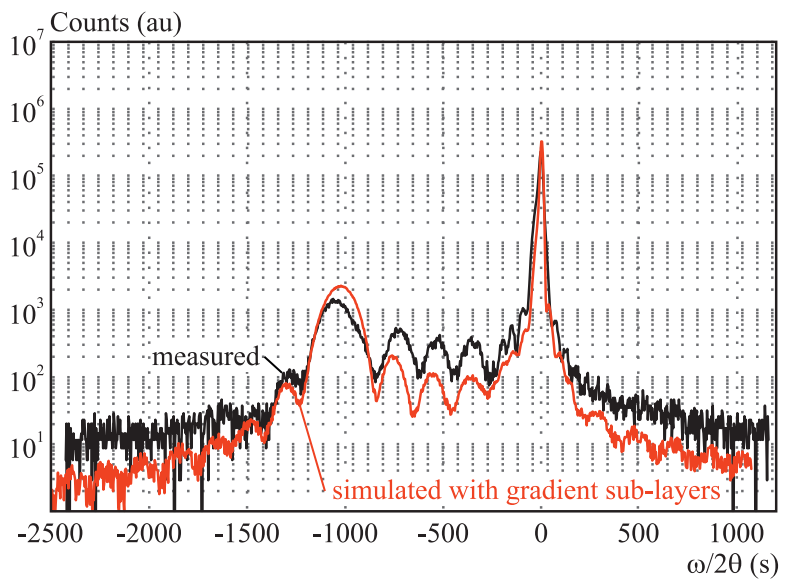

Fig. 9. The measured and simulated (004) $\omega / 2 \theta$ diffraction curves of the \#NI82SI $p^{+}$-GaInNAs/GaAs:Zn structure

Table 2. Structural parameters of the \#NI82 GaInNAs/GaAs structure with $101 \mathrm{~nm}$ thick, Zn doped $\left(2.3 \times 10^{18} \mathrm{~cm}^{-3}\right)$, doublegradient GaInNAs layer

\begin{tabular}{cccc}
\hline & $\operatorname{In}(\%)$ & $\mathrm{N}(\%)$ & $d(\mathrm{~nm})$ \\
\hline GaInNAs & 6.2 & & \\
$2^{\text {nd }}$ sublayer & $\uparrow$ & 0.1 & 80.0 \\
with linear gradient & 5.9 & & \\
\hline GaInNAs & 5.9 & 0.1 & \\
$\begin{array}{c}\text { Gt } \\
1^{\text {st }} \text { sublayer }\end{array}$ & $\uparrow$ & $\uparrow$ & 21.0 \\
with linear gradient & 5.0 & 0.26 & \\
\hline GaAs buffer & & $\sim 300$ \\
\hline \multicolumn{4}{c}{ GaAs substrate } \\
\hline \multicolumn{4}{c}{}
\end{tabular}

the doped structure. Summarizing, standard optical measurement methods do not provide sufficient, complementary to HRXRD measurements, information which could allow structural characterization of GaInNAs doped layers with gradient composition.

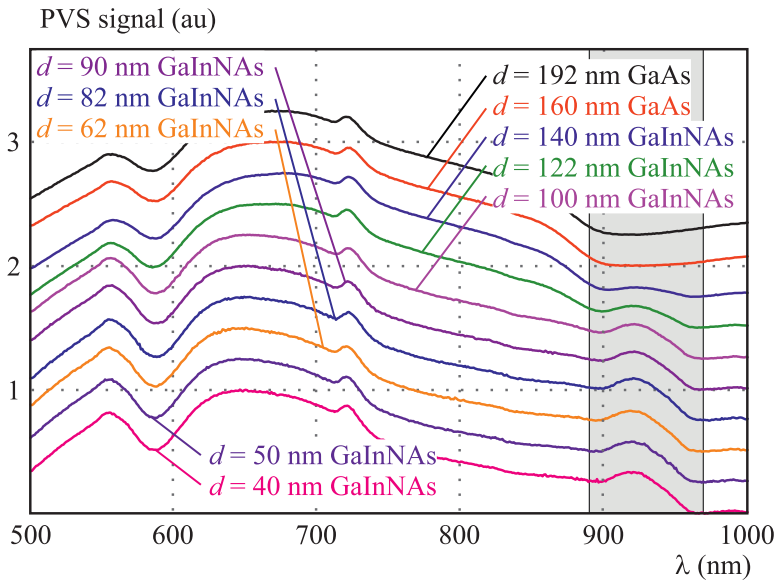

Fig. 8. PVS spectra of the \#NI105n p-GaInNAs/GaAs:Zn structure, with the uniform $\mathrm{p}$ type GaInNAs layer (carrier concentration varying in the range from 6 to $10 \times 10^{17} \mathrm{~cm}^{-3}$ ), measured at different depth of etching

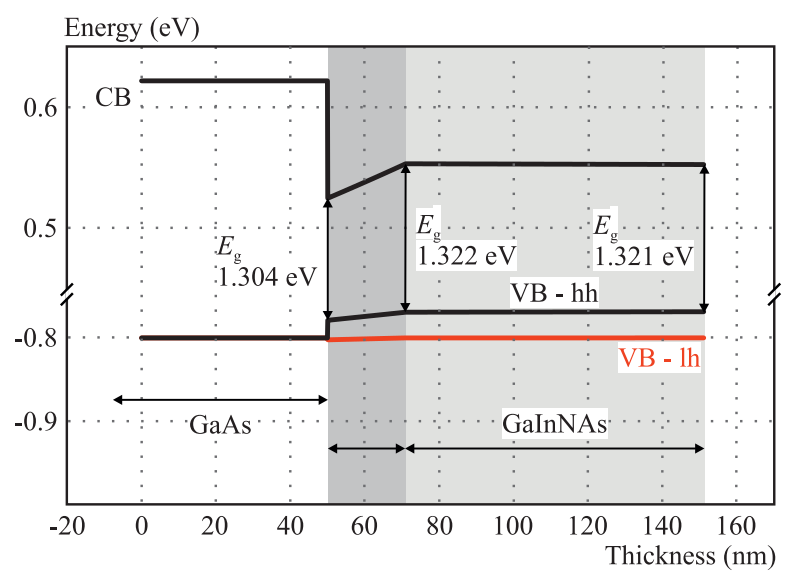

Fig. 10. Band alignment of the \#NI82 GaInNAs/GaAs structure with a $101 \mathrm{~nm}$ thick, $\mathrm{Zn}$ doped $\left(2.3 \times 10^{18} \mathrm{~cm}^{-3}\right)$, double-gradient GaInNAs layer

\section{PROPOSED SOLUTION}

For proper characterization of doped inhomogenous GaInNAs layers the HRXRD could be coupled with PVS measurements performed simultaneously with electrochemical capacitance-voltage (E-CV) profiling. By using the electrochemical profiler Bio-Rad PN4300 it is possible to measure the PVS signal of the GaInNAs layer coming from various depths of the layer. The PVS measurements of the investigated GaInNAs layers (Fig. 8) can be taken every $2 \mathrm{~nm}$ of etching, starting from the surface of the sample. The big advantage of this method is that the electrical response of the measured layer to the optical excitation depends on the depletion depth of the formed layer-electrolyte Schottky contact. The most important is that this depletion depth decreases with the doping level in the layer. It means that in the case of a highly doped layer the measured profile of the band-gap changes becomes more precise. If only the absorption edge shift would be visible along the GaInNAs layer (see the light gray area in Fig. 8) it could provide information about the change of the energy gap along the layer, which could 
be taken into consideration during HRXRD diffraction curves simulations as a gradient of composition. As a result, structural characterization of doped GaInNAs thick layers could be performed more accurately and more precise data, concerning the gradient of composition of GaInNAs doped layers, could be achieved.

Results of structural analysis of the thick doped $(2.3 \times$ $10^{18} \mathrm{~cm}^{-3}$ ) GaInNAs layer are shown in Figs. 9 and 10 and in Tab. 2. The presented $\omega / 2 \theta$ scan of the \#NI82 $\mathrm{p}^{+}$-GaInNAs/GaAs:Zn structure contains an asymmetrical distribution of oscillations, which indicates a gradient of the GaInNAs layer composition.

From the analysis of that structure the following structural data of the double-gradient GaInNAs:Zn were estimated (Tab. 2).

The calculated band alignment of the \#NI82 structure is presented in Fig. 10, where two linearly graded sublayers were distinguished. The valence bands for heavy holes and light holes are presented with the energy of the bandgap at the interfaces.

\section{CONCLUSION}

This work deals with the challenges of structural characterization of doped thick GaInNAs layers grown by atmospheric pressure metalorganic vapour phase epitaxy (APMOVPE). HRXRD is presented as an insufficient method for structural analysis of GaInNAs semiconductor compounds. The proposed algorithms of structural characterization of such materials, which couple HRXRD with BAC-based analysis of optical spectroscopy measurements, were faced with the examination of thick doped non-homogeneous GaInNAs layers. Photovoltaic spectroscopy performed during electrochemical capacitancevoltage profiling is meant to be used to support X-ray measurement analysis of such a kind of doped materials. Preliminary investigations of structural characterization of epitaxial structures with thick doped non-homogeneous GaInNAs layers were presented.

\section{Acknowledgement}

This work was co-financed by Wroclaw University of Technology statutory grant and Slovak-Polish International Cooperation Program no. SK-PL-0005-12

This paper summarizes in brief the essential issues of a work presented at ADEPT-2014, Advances in Electronics and Photonic Technologies, an international conference held in Tatranska Lomnica, High Tatras, Slovakia, on 1-4 June 2014

\section{REFERENCES}

[1] WEYERS, M.-SATO, M.-ANDO, H. : Red Shift of Photoluminescence and Absorption in Dilute GaAsN Alloy Layers, Japanese Journal of Applied Physics Part 231 No. 7A (1992), 853-855.

[2] BISPING, D.-PUCICKI, D.-FISCHER, M.-HOEFLING, S.-FORCHEL, A.: Influence of Arsenic Flux on The Annealing Properties of GaInNAs Quantum Wells for Long Wavelength
Laser Applications Around $1.6 \mu \mathrm{m}$, Journal of Crystal Growth 311 (2009), 1715-1718.

[3] KURTZ, S. R.-ALleRMAN, A. A.-JONES, E. D.-GEE, J. M.-BANAS, J. J.-HAMMONS, B. E. : InGaAsN Solar Cells with $1.0 \mathrm{eV}$ Band Gap, Lattice Matched to GaAs, Applied Physics Letters 74 No. 5 (1999), 729-731.

[4] PUCICKI, D.-BIELAK, K.-KUDRAWIEC, R.-RADZIEWICZ, D.-ŚCIANA, B.: Determination of Indium and Nitrogen Contents of InGaAsN Quantum Wells by HRXRD Study Supported by BAC Calculation of The Measured Energy Gap, Materials Science-Poland 31 No. 4 (2013), 489-494.

[5] LI, W.-PESSA, M.-LIKONEN, J.: Lattice Parameter in GaNAs Epilayerson GaAs: Deviation from Vegard's Law, Applied Physics Letters 78 No. 19 (2001), 2864-2866.

[6] ALBO, A.-CYTERMANn, C.-BAHIR, G.-FEKETE, D. : Utilizing The Interface Adsorption of Nitrogen or The Growth of High-Quality GaInAsN/GaAs Quantum Wells by Metal Organic Chemical Vapour Deposition for Near Infrared Applications, Applied Physics Letters 96 (2010), 141102.

[7] PUCICKI, D.-BIELAK, K.-CIANA, B.-RADZIEWICZ. D.-LATKOWSKA, M.-KOVÁČ, J.-VINCZE, A.-TLACZALA, M. : Determination of Composition of Non-Homogeneous GaInNAs Layers, In press.

Received 15 June 2014

Katarzyna Bielak (MSc, Eng) received the MSc degree in electronics from the Faculty of Microsystem Electronics and Photonics of Wrocław University of Technology (WUT), Poland, in 2012. Since October 2012 she has been a PhD student in WUT.

Karolina Żelazna (MSc, Eng) was born in Lubin, Poland, in 1989. She received the MSc degree in physics from the Faculty of Fundamental Problems of Technology, Wrocław University of Technology (WUT), Wrocław, Poland in 2013. During her studies, she had a half-year internship at the University of Würzburg in 2011, where she was engaged in the growth of semiconductor nanostructurs by molecular beam epitaxy. Currently, she is a PhD student at WUT.

Jarosław Seraficzuk (PhD, Eng) was born in Nysa, Poland, in 1978. He received the MSc degree in electronics from the Faculty of Electronics, Wrocław University of Technology (WUT), Wrocław, Poland, in 2002, and the PhD degree from the Faculty of Microsystem Electronics and Photonics, WUT, in 2006.

Andrej Vincze (PhD), born in 1975, graduated in Microelectronics from the Slovak University of Technology in Bratislava in 1999 and received his $\mathrm{PhD}$ degree in Electronics from the STU at 2006. He works at the International Laser Centre in Bratislava.

Łukasz Gelczuk (PhD, Eng) was born in Brzeg Dolny, Poland, in 1978. He received the MSc degree in microsystems from the Faculty of Microsystem Electronics and Photonics, Wroclaw University of Technology (WUT), Wrocaw, Poland, in 2002, and the PhD degree from the Faculty of Microsystem Electronics and Photonics, WUT, in 2006.

Piotr Dłużewski (PhD, Eng) was born in Warsaw, Poland, in 1953. He received the MSc from the Physics Faculty of Warsaw University in 1977, and $\mathrm{PhD}$ degree from the Institute of Physics, Polish Academy of Sciences, in 1990. Since 1998 he has been the team leader of Electron Microscopy Group of the Institute of Physics, Polish Academy of Sciences.

Biographies of other authors see on page 276 of this issue. 